\title{
Cold test Measurements of a BWO Slow-Wave Structure
}

\author{
W. Main, Y. Carmel, K. Ogura, J. Weaver and S. Watanabe \\ Laboratory for Plasma Research \\ University of Maryland, College Park, Maryland 20742
}

\begin{abstract}
Bead-on-string perturbation techniques have been primarily used to study particle accelerator cavities. These techniques are now extended to measure the dispersion relation and axial modes of several transverse magnetic (TM) modes in a slowwave structure with sinusoidally varying radius. This type of slow-wave structure is commonly used in high power backward wave oscillators (BWO). The dispersion relation is found from the discrete measured resonant frequencies and wave numbers of a cavity containing six periods of the slow-wave structure. The measured axial and radial field profiles are compared with the results of a $2-D$ code specially developed for calculation of the fields in these generators. For the first three TM modes the frequency error was less than $0.7 \%$.
\end{abstract}

\section{INTRODUCTION}

We present a study of a slow-wave structure which consists of a cylindrical waveguide with sinusoidally varying radius. A similar version of this structure was used in numerous $\mathrm{X}$-band relativistic high-power backward wave oscillator experiments $[1,2]$, however, until now there has not been a systematic analysis of its electromagnetic properties. The purpose of this study is twofold, first to experimentally determine the dispersion relation of the structure and second to test our numerical model of the fields inside the structure.

\section{Dispersion Relation}

A slow wave structure containing $\mathrm{N}$ periods will exhibit $N+1$ resonant frequencies associated with each transverse magnetic mode when shorted at planes of mirror symmetry. Discrete points on each branch of the dispersion relation are found by recording resonant frequencies $f_{r}$ and associated axial wave numbers $\beta_{r}$ for the set of axial modes associated with each transverse magnetic mode. Figure 1 shows the seven $\mathrm{TM}_{01}$ resonance peaks and derived dispersion relation for a six period slow wave cavity. The resonant frequencies are easily measured with a microwave network analyzer, but to find $\beta_{r}$ we must measure the axial field structure in the cavity. This is done hy perturbing the field near the axis of the cavity with a small metal bead. The resulting shift in resonant frequency is related to the $\mathrm{E}$ and $\mathrm{H}$ fields at the position of the bead. The number of axial variations in the field $n_{r}$ can be found by counting number of peaks in a plot of $\Delta f$ vs. axial bead position. For a structure with $\mathrm{N}$ periods of length $\mathrm{L}$ this condition for resonance can be stated as

$$
N L=\left(n_{r} / 2\right)(2 \pi / \beta)
$$

where $n_{r}$ is the number of half wavelengths along the axis of the structure. The axial wave number $\beta_{r}$ is then found from $n_{r}$ using the above equation. The complete dispersion relation can easily be constructed from these points. For convenience, the dispersion relation was measured on a "cold-test" structure built just for this purpose. This test structure had special end plates with access holes for positioning a bead; and an antenna for launching TM modes. We chose a cold-test structure with six axial periods, giving us seven points on our $\omega \beta$ diagram.

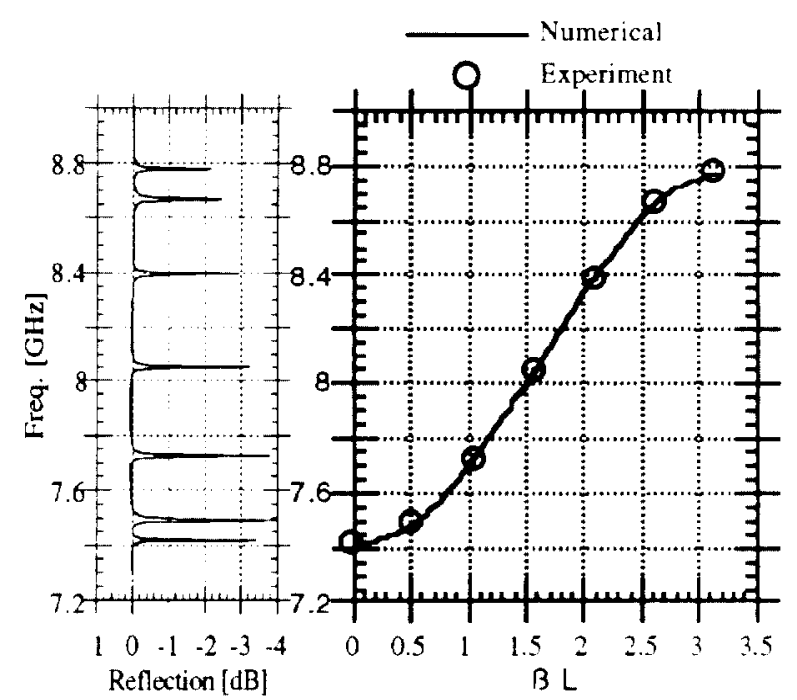

Figure 1. Left graph shows resonances as seen from a one-port measurement on a network analyzer. Right graph shows derived dispersion 
Experimental Verification of Numerical Model

Using the dispersion relation we can find the relation of beam energy and interaction frequency. However, to understand the more complex behavior of an intense beam in a slow wave structure we must use nonlinear particle codes. These codes require an accurate model of the fields in the structure. We use a model developed by Ogura [3] in which the fields are expanded in a spatially harmonic series, according to Floquet's theorem. Solving the dispersion relation for the sinusoidal boundary of the slow wave structure gives us the expansion coefficients. For computational reasons we truncate our expansions at nine terms.

Due to the complexity of this model it is important to experimentally verify the fields at various points in the cavity. Although we cannot directly measure the fields inside the cavity, we can test the numerical model by inserting the same perturbing object in the numerical model and in the experiment As a perturbing object we introduce a small conducting bead into the cavity. In the experiment, this bead is suspended by a small string, and the resulting frequency shift is measured with a network analyzer. In the numerical model we use perturbation theory to calculate the effect the bead has on the resonant frequency. For a small spherical bead, where the field is almost constant over $\Delta \mathrm{V}$, integration is unnecessary and the frequency perturbation is given by

$$
\frac{\Delta f_{r}}{f_{r}}=(2 \pi / 3) r_{o}^{3} 3\left(\frac{1}{2} H_{o}^{2}-E_{o}^{2}\right)
$$

where $E_{0}$ and $H_{0}$ are the field amplitudes normalized to the total field energy in the cavity. By comparing the measured and calculated frequency shift we can judge how well the numerical model agrees with experiment.

Since the only field component on axis is $E_{Z}$, the simplest test would be to perturb the field on the cavity axis. But, because of the difficulty in having both the bead and the antenna on axis, the bead was kept $0.543 \mathrm{~cm}$ away from the axis. At this radius it was still possible to find $k_{Z}$ for the $\mathrm{TM}_{01}$ modes by counting peaks in the frequency shift.

\section{EXPERIMENTAL ARRANGEMEN'T}

Three components were required to measure the cavity properties: the mode launcher, the network analyzer and the perturbing object. Figure 2 presents a schematic diagram of the measurement apparatus. The dimensions of the system appear in Table I. The launcher was a simple axial wire which could be moved in and out of the cavity to adjust the coupling. Before making measurements the reflection from the antenna was stored in memory and subtracted from subsequent measurements. This procedure made the resonances easier to identify.

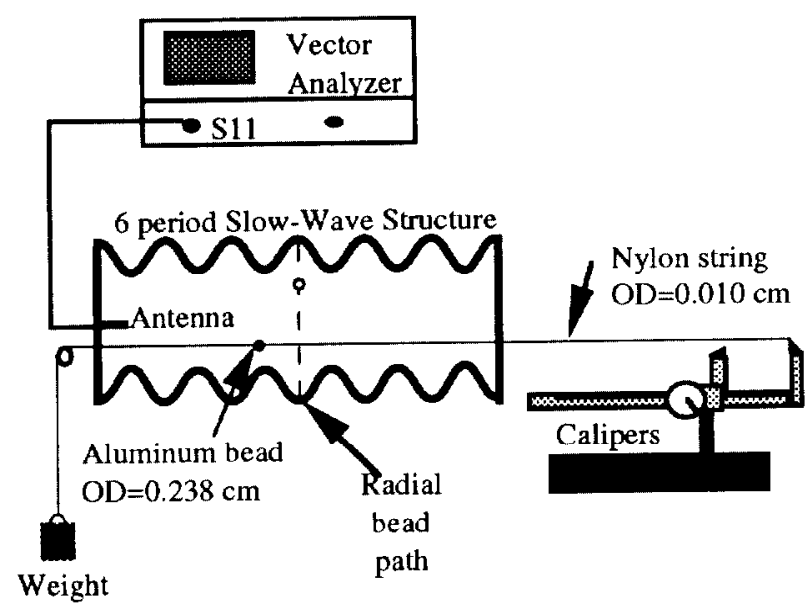

Figure 2. Schematic diagram of measurement apparatus

Table I Dimensions of slow wave structure.

$\begin{array}{ll}\text { Structure Period } & 1.667 \mathrm{~cm} \\ \text { Total Length } & 10 \mathrm{~cm}(6 \text { periods }) \\ \text { Radius }[\mathrm{cm}] & 1.5+.41 \operatorname{Sin}(3.7 \mathrm{z}) \\ \text { Bead diameter } & 0.239 \mathrm{~cm} \\ \text { string at radius } & 0.543 \mathrm{~cm}\end{array}$

The perturbing object used for the majority of this work was an $0.239 \mathrm{~cm}$ OD spherical aluminum bead. The bead was suspended by a $0.010 \mathrm{~cm}$ OD nylon thread which was connected to the bead through a $0.033 \mathrm{~cm}$ ID hole on the bead's axis. Four sets of access holes were drilled in the cavity for the nylon thread. One parallel to the axis of the cavity, at a radius of $0.543 \mathrm{~cm}$ and three in the radial direction at axial positions corresponding to the maximum, average and minimum radii. The bead was positioned 
in the cavity by connecting one end of the thread to a dial caliper. The other end of the thread was heal taught by a small weight. The effect of the nylon string on the resonant frequency was less than $500 \mathrm{KHz}$ in all cases.

\section{RESULTS}

\section{Dispersion Relation}

The dispersion relation results appear both as tables and plots of $f_{r}$ and $\beta_{r}$. All measurements are compared with numerical calculation. The dispersion measurements agree very well with the boundary specific calculation of Ogura and the general boundary calculation using the code Superfish [4]. The experimental data for the $\mathrm{TM}_{01}$ mode were on average, $0.17 \%$ higher than Ogura's results and $0.03 \%$ lower than Superfish, and for the $\mathrm{TM}_{02}$ mode the experimental data were $0.05 \%$ Iower than Ogura's results. Superfish data was not available for the $\mathrm{TM}_{02}$ modes.

Graphical comparison of the experimental results with Superfish were presented in Figure 1. This figure shows the dispersion curve and reflection data with a common frequency axis. The Superfish results, which were calculated as 7 points are presented as a smooth curve to show the form of the curve and aid in comparing with experiment. In the $\mathrm{TM}_{02}$ case we were not able to experimentally excite the $\pi$-mode, although Ogura's code did find this resonance.

\section{Field Calculation}

Calculations were made for each of seven axial modes on three TM modes. For each of these field structures we calculated the frequency perturbation along the axis and along a radius. We present the results of the cavity field calculation in several forms for the $\mathrm{TM}_{01} 2 \pi / 3$ mode. To help visualize the field structures we present plots of the field lines (Fig. 3a) along with the axial (Fig 3b) and radial (Fig 3c) frequency perturbation plots. The perturbation plots are only used to compare the numerical and experimental results. In these plots the magnitude of the frequency perturbation should not be mistaken to be proportional to the magnitude of the field squared. This is only true when either the $\mathrm{E}$ or $\mathrm{H}$ field dominates, i.e. on the axis of the structure where $\mathbf{H}=0$. (a)
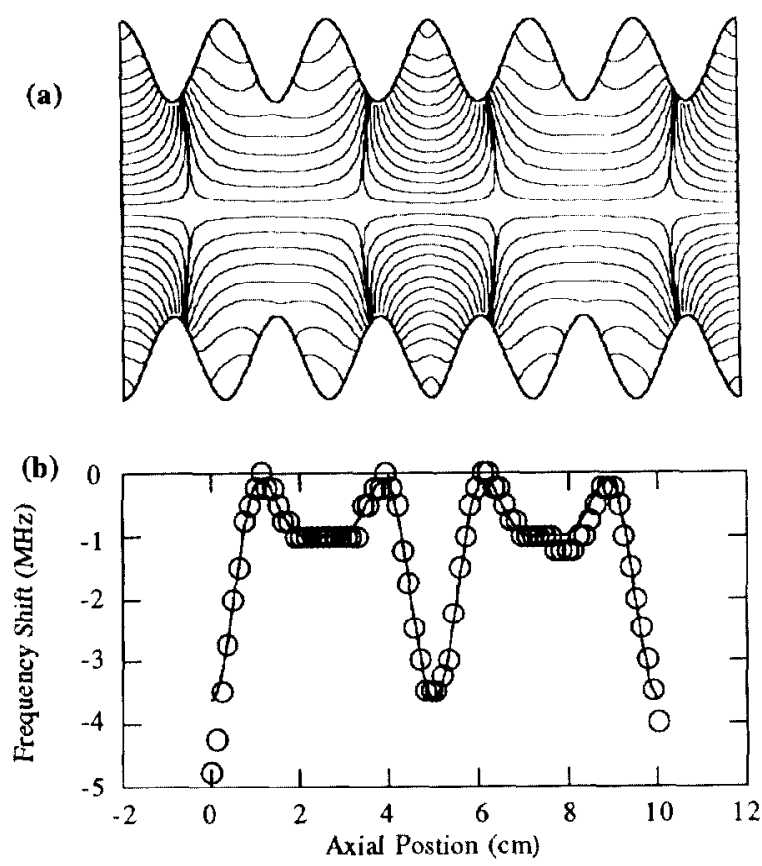

(c)

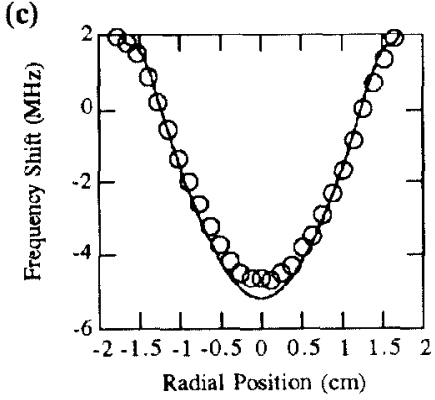

Figure 3. Results for the $\mathrm{TM}_{01} 2 \pi / 3$ mode. (a) Field lines produced by numerical model (Ogura), (b) comparison of measured and calculated field perturbations for axial bead traverse and (c) radial bead traverse.

\section{REFERENCES}

1. N.F. Kovalev et al, JETP Letters, 18, pp138-140, (1973)

2. R.A. Case et al, "High Power BWO driven by a Relativistic Electron Beam", IEEE Trans. Plasma Sci. PS-13 pp. 559-562 (1985)

3. W.Main, Y. CarmeI, J. Weaver, K. Ogura,"The Electromagnetic Properties of Finite Length Slow-Wave Structures for Intcraction with a Relativistic Electron Beam," IEEE Trans. MTT Submitted.

4. K. Halbach, R. Holsinger, Proceedings, Particle Accelerator Conference pp. 213 (1976) 